\title{
Breath-powered sumatriptan dry nasal powder: an intranasal medication delivery system for acute treatment of migraine
}

This article was published in the following Dove Press journal:

Medical Devices: Evidence and Research

\author{
Stewart J Tepper' \\ Merrilee R Johnstone ${ }^{2}$ \\ 'Department of Neurology, Geisel \\ School of Medicine at Dartmouth, \\ Hanover, NH, USA, ${ }^{2}$ Prescott Medical \\ Communications Group, Chicago, IL, \\ USA
}

\begin{abstract}
There is a need for fast-acting, non-oral medication options for migraine because some attacks develop rapidly and some are accompanied by nausea, vomiting, and gastroparesis, which can hinder oral medication uptake and absorption. The most commonly prescribed migraine medications are oral triptans, with sumatriptan as the most common. However, oral triptans are associated with adverse events (AEs) of atypical sensations that may be problematic for patients. Subcutaneous (SC) injectable sumatriptan and conventional liquid triptan nasal spray formulations are also available, but the frequency of atypical sensations is the highest with SC sumatriptan, and the intense bitter taste of conventional liquid triptan nasal spray discourages use. AVP-825 (ONZETRA ${ }^{\circledR}$ Xsail $^{\circledR}$ ) is an intranasal medication delivery system containing 22 $\mathrm{mg}$ sumatriptan nasal powder that is now available in the USA for the acute treatment of migraine with or without aura in adults. The objective of this review is to summarize the development of AVP-825, which utilizes unique features of nasal anatomy to achieve efficient absorption and reduced systemic exposure. Literature searches for "sumatriptan nasal powder", "AVP-825", and "sumatriptan intranasal" were conducted. Review articles and pharmacokinetic, Phase II and Phase III studies were evaluated. AVP-825 demonstrates an earlier onset of efficacy and lower rate of atypical sensations than the oral standard of care, which can be attributed to its fast absorption and low systemic exposure. AEs of abnormal taste are predominantly mild. These results confirm the initial design concept for AVP-825, which aligned pharmacokinetics, anatomy, and drug presentation in a novel device to achieve optimal outcomes for the acute treatment of migraine.
\end{abstract}

Keywords: migraine, triptan, sumatriptan, AVP-825, intranasal, Onzetra

\section{Introduction}

\section{Migraine: a headache disorder}

Migraine is a primary headache disorder characterized by attacks of moderate-tosevere headaches, typically on one side of the head, which have a pulsating quality, are worsened by physical activity, and are associated with nausea and/or vomiting, photophobia, and phonophobia. ${ }^{1,2}$ In up to a third of patients, migraines are preceded or accompanied by aura, which most commonly consists of visual symptoms. ${ }^{1,3}$ In the most recent Global Burden of Disease Study, migraine was the sixth most prevalent disorder and the second leading cause of years lived with disability worldwide. ${ }^{4}$ In a recent US survey, $20.0 \%$ of women and $9.7 \%$ of men aged $\geq 18$ years had a severe headache or migraine within the past 3 months. ${ }^{5}$ Beyond the reduction in disability that would accompany successful treatment of individual migraine attacks, improved treatment may also reduce the risk of chronic migraine development. ${ }^{6}$
Correspondence: Stewart J Tepper Neurology Department, DartmouthHitchcock Medical Center, I Medical Center Drive, Lebanon, NH 03756 USA $\mathrm{Tel}+\mathrm{I} 6036505104$

$\mathrm{Fax}+\mid 603653$ I273

Email Stewart.J.Tepper@Dartmouth.edu 


\section{Migraine medication: the importance of formulation and delivery route}

The current mainstays of migraine treatment are orally administered over-the-counter pain relievers (acetaminophen, nonsteroidal anti-inflammatory drugs) and prescription triptans (serotonin $[5-\mathrm{HT}]_{1 \mathrm{~B} / \mathrm{D}}$ receptor agonists). ${ }^{7,8}$ Oral medications are easy to use but can have drawbacks, particularly for migraine patients. For example, those who experience nausea and/or vomiting with their migraines may have difficulty taking or retaining oral medication. ${ }^{9,10}$ In addition, gastroparesis (delayed emptying of the stomach) may occur in migraine patients during or between migraines and appears to reduce absorption, consistency, and effectiveness of oral medications. ${ }^{9,11,12}$ Oral medications are also subject to gastrointestinal (GI) and hepatic first-pass metabolism, which can change their pharmacokinetics and clinical effects. For example, orally administered sumatriptan tablet, the most commonly prescribed triptan, is largely converted to an inactive metabolite through first-pass metabolism and, combined with incomplete absorption, has a bioavailability of about $15 \%$ in humans. ${ }^{13,14}$ Notably, one-third of patients in clinical trials fail to obtain headache relief with sumatriptan oral. ${ }^{15}$

While there are a limited number of ways of bypassing the GI tract, non-oral formulations of some triptans are available and primarily include subcutaneous (SC) injection of sumatriptan (needle and needle-free) and liquid intranasal triptans (sumatriptan and zolmitriptan). SC sumatriptan injection is fast-acting but has a substantial rate of injectionsite reactions and triptan-associated atypical sensations (eg, tingling, numbness, hot sensation, flushing, and chest/jaw/ neck tightness), and fewer than $10 \%$ of eligible migraine patients use SC sumatriptan for treatment. ${ }^{16-21}$ Intranasal triptans are also fast-acting and effective with different nasal delivery methods available. This review highlights the nose as a medication delivery route and currently available intranasal delivery methods for migraine, including the Breath Powered ${ }^{\circledR}$ sumatriptan dry nasal powder, AVP-825, medication delivery system approved by the US Food and Drug Administration as ONZETRA ${ }^{\circledR}$ Xsail $^{\circledR}$ (sumatriptan nasal powder; Avanir Pharmaceuticals, Inc. Aliso Viejo, CA) for the acute treatment of migraine with or without aura in adults. ${ }^{22}$

\section{The nose as a route for drug delivery}

The nose is an attractive delivery route for medication, as it has a large mucosal surface area and is highly vascular, making it well suited for rapid absorption of drugs into the systemic circulation. ${ }^{23,24}$ As the primary purpose of the nasal airway is to protect the lungs from hazardous exposure, some aspects of nasal anatomy and physiology present obstacles to efficient nasal drug delivery. ${ }^{23}$ The richly vascular mucosa most conducive to drug absorption lies in the upper posterior nose, beyond the narrow nasal valve. ${ }^{23,24}$ The small dimensions of the nasal valve and its triangular shape that narrows further during nasal inhalation are impediments to drug delivery beyond it. ${ }^{23}$

\section{Conventional liquid nasal sprays}

Intranasal drug delivery commonly has been accomplished using liquid nasal spray delivered through a metered-dose mechanical pump, ${ }^{23}$ which is the case for the conventional liquid intranasal sumatriptan formulation. Activating the spray pump propels liquid medication outward in a hollow spray cone, and even if the spray pump is inserted deep into the nostril, there is a mismatch between the geometry of the circular spray cone and the narrow triangular nasal valve opening. ${ }^{23}$ Imaging studies using a liquid solution of radiolabeled diethylene triamine pentaacetic acid (DTPA) show that deposition from a conventional liquid intranasal spray pump largely occurs anterior to the nasal valve and on the interior floor of the nose, with only a limited portion of the liquid penetrating beyond the nasal valve to the highly absorptive upper posterior nasal cavity (Figure 1A and C) ${ }^{23-25}$ Pharmacokinetic studies of conventional sumatriptan liquid nasal spray indicate that a substantial portion of the dose administered through conventional liquid spray pump is subsequently swallowed. ${ }^{24-28}$ Once swallowed, the medication becomes, in effect, an orally administered medication that is subject to the disadvantages noted previously. In addition, bitter taste is often reported with these nasal sprays because bitter-sensing taste buds at the base of the tongue are exposed to the liquid medication. ${ }^{19,24}$

\section{Breath-powered exhalation delivery of sumatriptan dry nasal powder:AVP-825}

AVP-825 was designed to improve intranasal medication delivery by taking greater advantage of nasal anatomy to achieve more effective drug delivery to the upper posterior absorptive surfaces of the nasal cavity. ${ }^{22,24}$ AVP- 825 consists of a breath-powered exhalation device and a low-dose dry powder formulation of sumatriptan (22 $\mathrm{mg}$ ), supplied as a reusable mouthpiece and two disposable nosepieces each containing a capsule filled with $11 \mathrm{mg}$ sumatriptan nasal powder (Figure 2). A dry powder formulation has several potential advantages over liquid for intranasal delivery, including greater stability, reduced need for preservatives, and greater adherence to the upper posterior nasal mucosa. ${ }^{23}$ To use AVP-825, the user inserts a nosepiece into the device, 

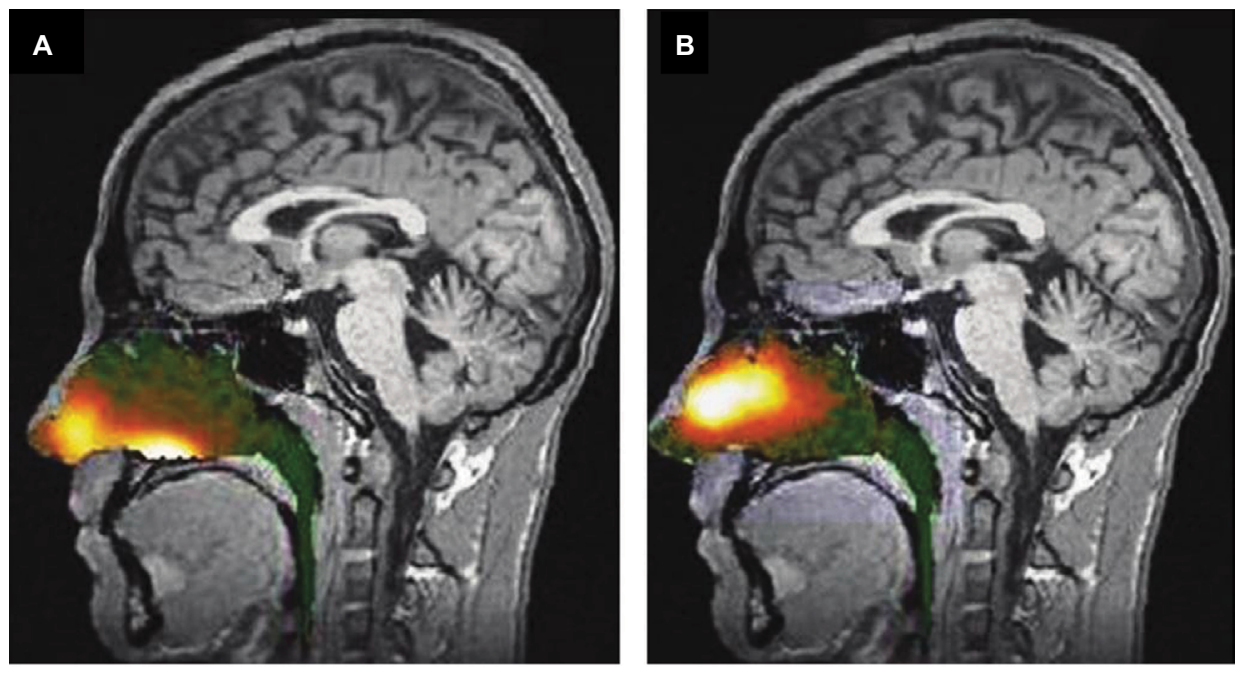

C

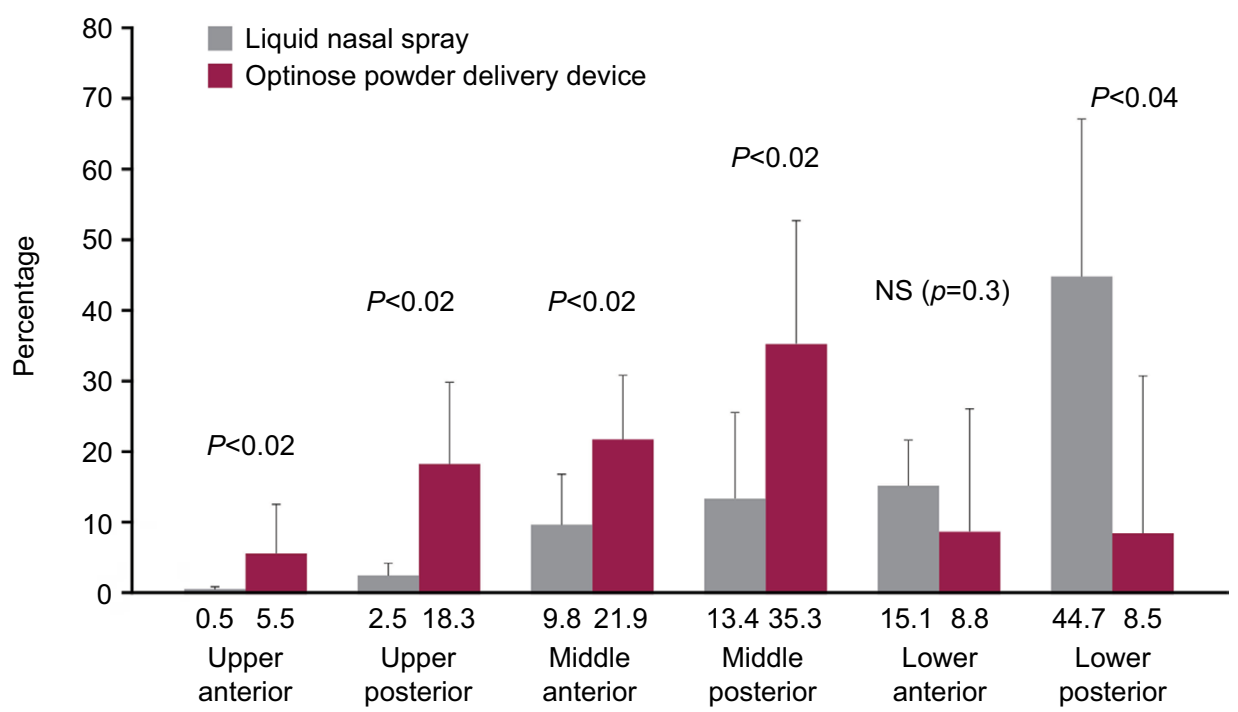

Figure I Imaging of intranasal delivery of (A) conventional liquid spray (radiolabeled diethylene triamine pentaacetic acid) vs (B) powder (radiolabeled lactose) with the breath-powered device and $(\mathbf{C})$ quantification of deposition patterns.

Notes: Gamma camera image information (logarithmic hot iron intensity scale) from the nasal cavity is superimposed on the corresponding sagittal magnetic resonance imaging section. The image represents deposition 2 minutes after delivery. The images were from the same subject after each method of administration, which were representative of the overall findings from the seven patients included in the study. Panel C shows the percentage distribution by nasal region 0-2 minutes after administration (bars are mean \pm SD). Republished with permission of Mary Ann Liebert Inc., from Djupesland PG, Skretting A. Nasal deposition and clearance in man: comparison of a bidirectional powder device and a traditional liquid spray pump. J Aerosol Med Pulm Drug Deliv. 2012;25(5):280-289 permission conveyed through Copyright Clearance Center, Inc. ${ }^{25}$

presses a button to pierce the medication capsule, and then slides the nosepiece into one nostril until it forms a seal with the nostril opening. This action expands the nasal valve. The user then rotates the device to place the mouthpiece into the mouth and blows into it, which leads to closing of the soft palate and further expansion of the nasal passage (Figure $3 \mathrm{~A})$. The closing of the soft palate isolates the nasal cavity from the throat, which prevents medication deposition into the throat, lungs, or GI tract. In addition, the positive pressure created by blowing into the device allows airflow to enter one nostril, diffuse within the upper nasal cavity, and exit through the other nostril, resulting in Bi-Directional ${ }^{\circledR}$ delivery (Figure 3B). The user then repeats the process with the second nosepiece in the opposite nostril. Each nosepiece delivers an average dose of 7.5-8.1 mg sumatriptan, providing a total dose of 15-16.2 mg sumatriptan per treatment episode from two nosepieces. ${ }^{22}$

Delivery of powder (radiolabeled lactose) through the breath-powered exhalation technology yields significantly greater deposition in the upper posterior, highly absorptive regions of the nasal cavity beyond the nasal valve (Figure 1B and C) compared to conventional liquid spray (radiolabeled 
2 reusable blue devices ( 1 for immediate use +1 spare)

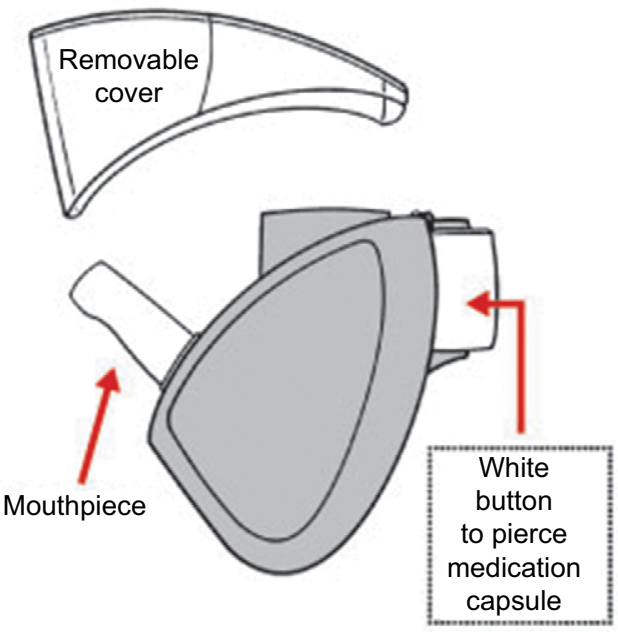

Disposable nosepieces (in foil pouches)

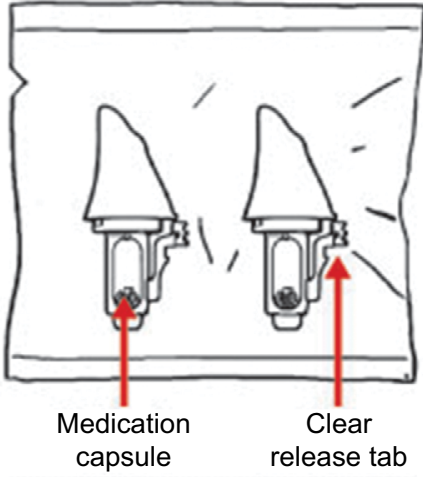

1 dose $=2$ nosepieces

(1 for each nostril)

Each disposable white

nosepiece contains half a dose

of medication

(11 mg of sumatriptan).

A full dose is $22 \mathrm{mg}$.

Figure 2 AVP-825 for intranasal delivery of sumatriptan powder.

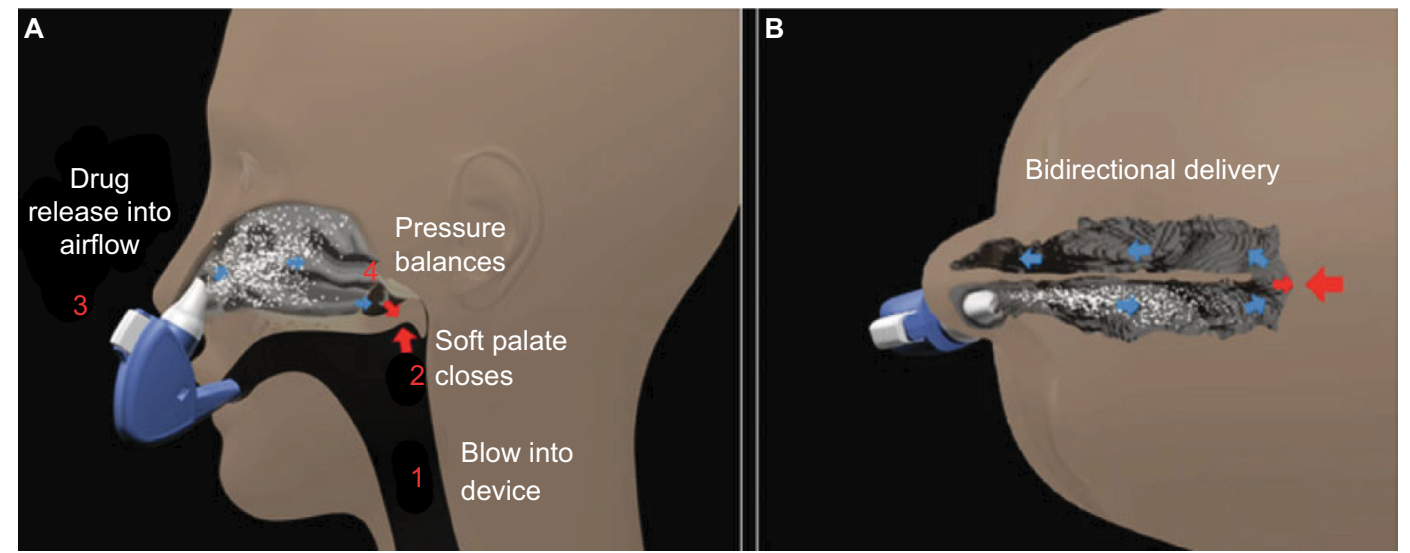

Figure 3 Illustration of breath-powered intranasal delivery.

Note: (A) Lateral view showing breath powered mechanism and (B) superior view showing bi-directional mechanism. In both panels, blue arrows indicate air flow and red arrows indicate the closing of the soft palate (large arrow) and pressure balance (small arrow). Copyright (C20I3. John Wiley and Sons. Reproduced from Djupesland PG, Messina JC, Mahmoud RA. Breath powered nasal delivery: a new route to rapid headache relief. Headache. 2013;53(Suppl 2): 72-84.24

DTPA) (Figure 1A and C), as shown by imaging studies. ${ }^{23-25}$ Pharmacokinetic data comparing AVP-825 and conventional sumatriptan liquid nasal spray (20 mg) support these imaging studies, as AVP-825 shows faster absorption, with an earlier and significantly higher peak plasma concentration and a $61 \%$ higher systemic sumatriptan exposure in the first 30 minutes (Figure 4A), despite a 20\% lower delivered dose. ${ }^{29}$ AVP-825 also produces faster sumatriptan absorption compared to $100 \mathrm{mg}$ sumatriptan oral tablet (Figure 4B) and a lower peak and total exposure than the $100 \mathrm{mg}$ oral tablet or $6 \mathrm{mg}$ SC sumatriptan injection, ${ }^{29,30}$ indicating the possibility of improved tolerability with AVP-825.

\section{Evaluating AVP-825 efficacy and safety in clinical trials}

AVP-825 was evaluated as an acute treatment of migraine in one Phase II and two Phase III randomized, double-blind, controlled trials in adults. ${ }^{31-33}$ Two of these trials compared AVP-825 to placebo in a single migraine attack (the Phase II study $^{32}$ and the Phase III TARGET study [NCT01462812] ${ }^{31}$ ), and one trial compared AVP-825 to $100 \mathrm{mg}$ sumatriptan oral tablet (well-established standard of care) over the course of multiple migraine attacks, a randomized, controlled comparative efficacy trial (The COMPASS study, NCT01667679). ${ }^{33}$ In these trials, AVP-825 demonstrated superior efficacy 

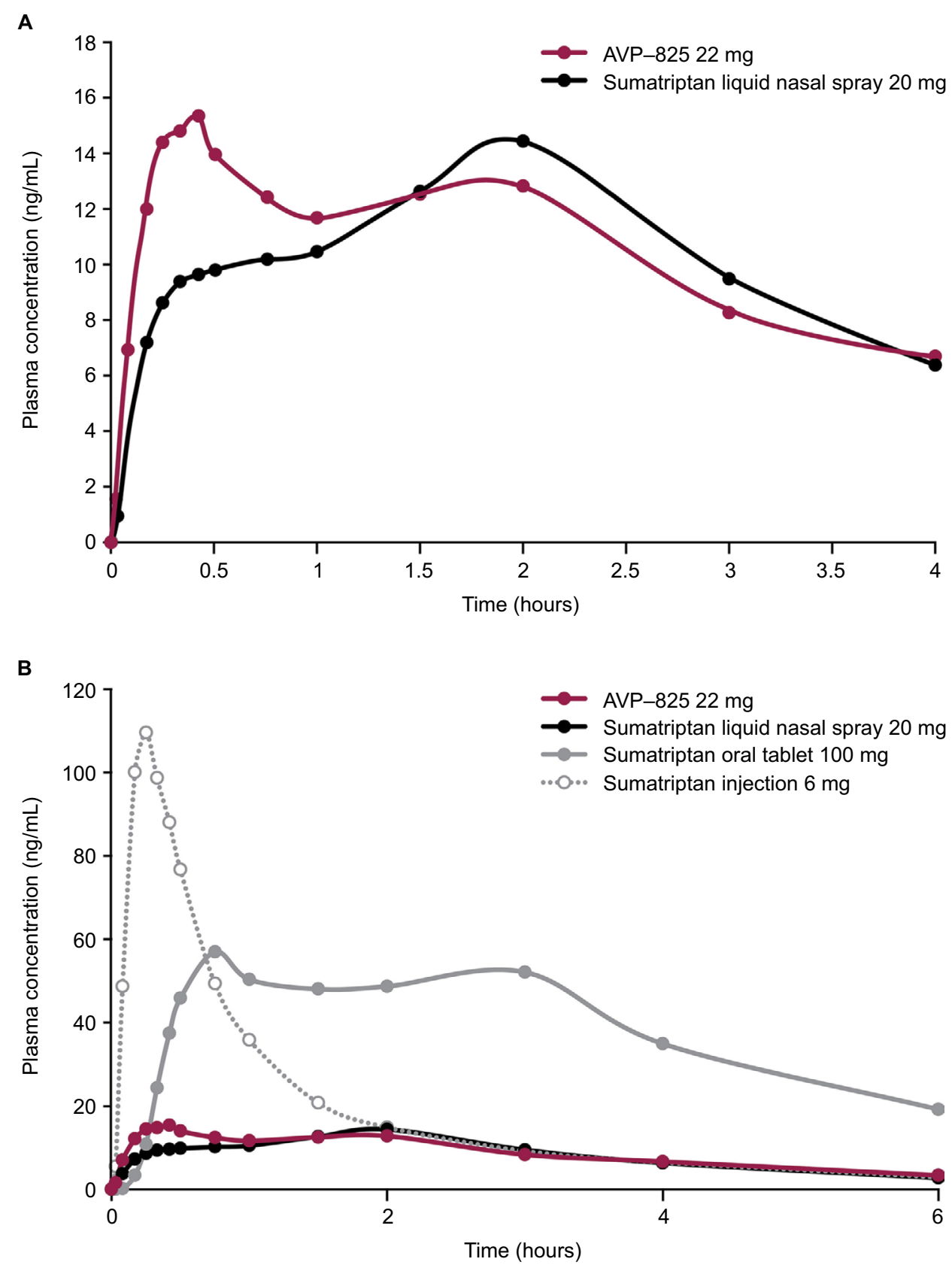

Figure 4 Sumatriptan plasma concentration-time profiles over (A) the first 4 hours after AVP-825 $(22 \mathrm{mg})$ and conventional sumatriptan liquid nasal spray (20 mg) and (B) the first 6 hours of the entire 14-hour sampling period for AVP-825, conventional sumatriptan liquid nasal spray $(20 \mathrm{mg})$, sumatriptan oral tablet (100 mg), and subcutaneous sumatriptan injection $(6 \mathrm{mg})$.

Note: Copyright @2013. John Wiley and Sons Inc. Adapted from Obaidi M, Offman E, Messina J, Carothers J, Djupesland PG, Mahmoud RA. Improved pharmacokinetics of sumatriptan with Breath Powered ${ }^{\mathrm{TM}}$ nasal delivery of sumatriptan powder. Headache. 2013;53(8):1323-1333..29

compared to placebo and an earlier onset of efficacy compared to $100 \mathrm{mg}$ sumatriptan oral tablets. Advantages on other measures of efficacy (eg, rates of nausea, photophobia, and phonophobia, assessment of disability, and patient-assessed meaningful relief) were also observed. AVP-825 treatment was well tolerated in all three trials, with no serious adverse events (AEs) and few discontinuations due to AEs. The most common AEs in all trials related to the site of administration were predominantly mild in severity, and AEs related to abnormal taste did not lead to any discontinuations. ${ }^{34}$ There was only one case of a mild atypical sensation (paresthesia) in the single-attack Phase II and III placebo-controlled trials, and in the multiattack Phase III COMPASS trial, atypical sensations occurred at a significantly lower rate with AVP$825 \mathrm{vs} 100 \mathrm{mg}$ sumatriptan oral tablet.

The prospect of a device that could efficiently deliver low-dose sumatriptan led to the initial design of AVP-825. The results were quicker onset of efficacy of sumatriptan 
compared to the oral formulation with lower systemic exposure and fewer side effects, confirming the design concepts and the pharmacokinetic promise.

\section{Phase II study $(\mathrm{N}=117)$}

Two doses of sumatriptan dry nasal powder (11 and $22 \mathrm{mg}$ ) delivered through the breath-powered exhalation system were compared to an identical placebo delivery system in the treatment of a single migraine attack of moderate-to-severe intensity. ${ }^{32}$ After treatment, a significantly greater proportion of patients in each sumatriptan nasal dry powder dose group (11 and $22 \mathrm{mg}$ ) vs placebo were pain free at 120 minutes (54\% and $57 \%$ vs $25 \%, P<0.05$, primary end point), had pain relief as early as 60 minutes ( $73 \%$ and $74 \%$ vs $38 \% ; P<0.01)$, and had pain relief at 120 minutes ( $84 \%$ and $80 \%$ vs $44 \%$, $P<0.001$ and $P<0.01)$. Efficacy was sustained as both sumatriptan nasal dry powder dose groups showed significantly greater rates of sustained pain freedom from 120 minutes up to 48 hours compared to placebo ( $47 \%$ and $49 \%$ vs $22 \%$, $P<0.05)$. AEs occurred in $18 \%$ and $23 \%$ of patients in the 11 and $22 \mathrm{mg}$ sumatriptan nasal powder groups, respectively, vs $5 \%$ for placebo, and the most common AE was bitter or metallic taste $(10 \%$ and $13 \%$ vs $0 \%)$. No events of chest discomfort or pain, paresthesia, or asthenia were reported for the active treatment groups, and there were no serious AEs or withdrawals due to AEs.

\section{Phase III TARGET study $(\mathrm{N}=230)$}

AVP-825 (22 mg) was compared to an identical placebo delivery system (containing lactose powder) in the treatment of a single migraine attack of moderate-to-severe intensity. ${ }^{31}$ A significantly greater proportion of patients treated with AVP-825 vs placebo were pain free at 120 minutes $(34.3 \%$ vs $17.3 \%, P=0.008)$, had pain relief as early as 30 minutes (41.7\% vs $26.9 \% ; P=0.03$ ), and had pain relief at 120 minutes (67.6\% vs $45.2 \%, P=0.002$, primary end point). Efficacy was sustained as AVP-825 showed a significantly greater rate of sustained pain freedom from 120 minutes up to 48 hours compared to placebo $(20.4 \%$ vs $8.7 \%, P=0.02)$. The most common AEs ( $\geq 2 \%$ in any treatment group) were abnormal product taste (22\% AVP-825 vs $4 \%$ placebo), nasal discomfort ( $13 \%$ vs $2 \%$ ), rhinorrhea ( $5 \%$ vs $3 \%$ ), and rhinitis $(3 \%$ vs $0 \%$ ). One patient reported mild paresthesia with AVP-825, but there were no other reports of atypical sensations. There were no serious AEs, and only one patient withdrew because of AEs (placebo treatment).

\section{Phase III COMPASS study $(\mathrm{N}=275)$}

AVP-825 (22 mg) was compared to $100 \mathrm{mg}$ sumatriptan oral tablet using a double-dummy, crossover trial design in the treatment of multiple migraine attacks (Figure 5). ${ }^{33}$ Treatment began within 1 hour of the onset of the migraine attacks, even if pain was mild. Patients treated up to five migraines

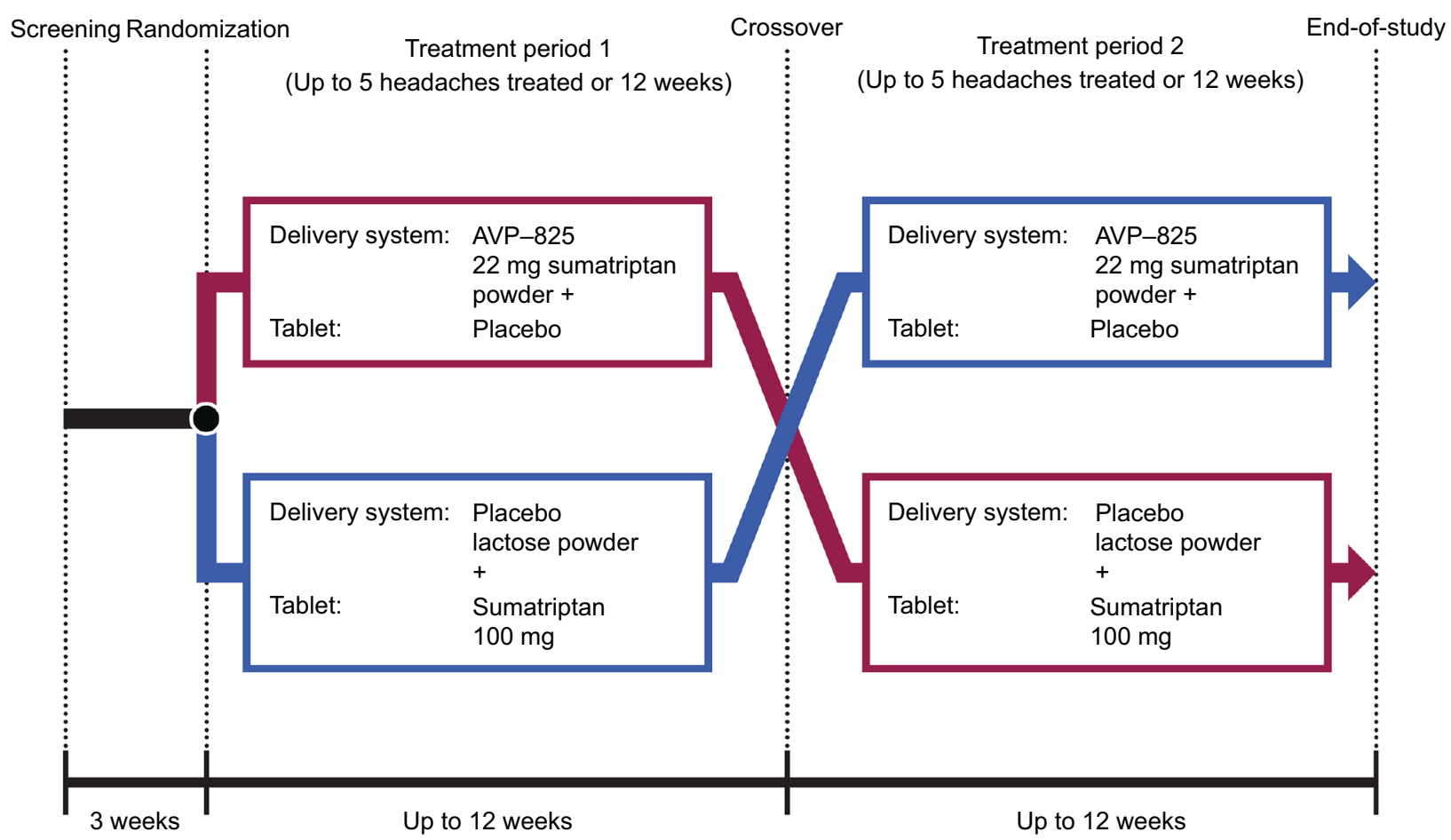

Figure 5 COMPASS study design.

Note: Reproduced from Silberstein S, Winner PK, McAllister PJ, et al. Early onset of efficacy and consistency of response across multiple migraine attacks from the Randomized COMPASS Study: AVP-825 Breath Powered ${ }^{\circledR}$ Exhalation Delivery System (Sumatriptan Nasal Powder) vs Oral Sumatriptan. Headache. 20I7;57(6):862-876. (C) 2017 American Headache Society. ${ }^{34}$ 
per treatment period (up to 12 weeks) with AVP-825 plus oral placebo tablet or an identical placebo device (containing lactose powder) plus $100 \mathrm{mg}$ sumatriptan oral tablet for the first double-blind treatment period and then switched treatment for the next period. Overall, $>1,500$ migraine attacks were evaluated.

A significantly greater reduction in migraine pain intensity occurred with AVP-825 treatment vs sumatriptan oral in the 30 minutes postdose, as assessed by summed pain intensity differences (SPID-30, 10.80 vs $7.41, P<0.001$, primary end point), and this was true regardless of whether attacks were treated when pain was mild or moderate/severe. ${ }^{34}$ As early as 15 minutes postdose, rates of pain freedom (7.2\% vs $3.7 \%, P=0.008)$ and pain relief $(27.9 \%$ vs $20.9 \%$, $P=0.007)$ were significantly greater with AVP- 825 vs sumatriptan oral, and significant differences in favor of AVP-825 continued through every time point up to and including 90 minutes postdose. Sustained efficacy was similar between treatments, as measured by rates of sustained pain relief and sustained pain freedom from 120 minutes up to 48 hours. AEs occurred in $53.9 \%$ and $32 \%$ of patients for AVP- 825 and $100 \mathrm{mg}$ sumatriptan oral tablet, respectively, with local administration site AEs such as abnormal taste (26\% vs $4 \%$ ) and nasal discomfort (16\% vs $1 \%$ ) driving the difference in overall AE rate.$^{34}$ However, these AEs were predominantly mild in severity (Figure 6) and led to few discontinuations (one patient each with nasal discomfort, throat irritation, and throat tightness). ${ }^{33,34}$ The rate of atypical sensations was significantly lower with AVP-825 compared to $100 \mathrm{mg}$ sumat- riptan oral tablet ( $2 \%$ vs $5 \%, P=0.02)$, and throughout the trial, no serious AEs occurred, and $<2 \%$ of patients receiving either treatment withdrew because of AEs.

\section{Expert opinion Development of AVP-825: from bench to medication delivery}

Early efficacy and tolerability are important aspects of patient satisfaction with migraine therapy. ${ }^{35-37}$ A key advantage of AVP-825 is that it allows for quick drug absorption and thereby an earlier onset of action relative to typical oral migraine therapies. In addition, AVP-825 delivers a lower sumatriptan dose relative to sumatriptan oral, which results in lower systemic exposure and a correspondingly lower incidence of atypical sensations. Based on clinical experience with migraine patients who have experienced triptanassociated atypical sensations and the resulting negative impact on compliance and tolerability, we argue that this difference is clinically important and should be considered in the treatment decision-making process.

For migraine, which can be accompanied by nausea, vomiting, and gastric stasis, a non-oral medication option such as AVP-825 can be optimal. Furthermore, while nausea is often a symptom of migraine, it can also be a result of treatment. ${ }^{38,39}$ Recent longitudinal modeling analyses of data from the COMPASS trial explored the impact of treatment on nausea and showed that AVP-825 led to more rapid early reductions in nausea rates during the first hour after treatment, reduced odds of nausea from 30 minutes to 2 hours following

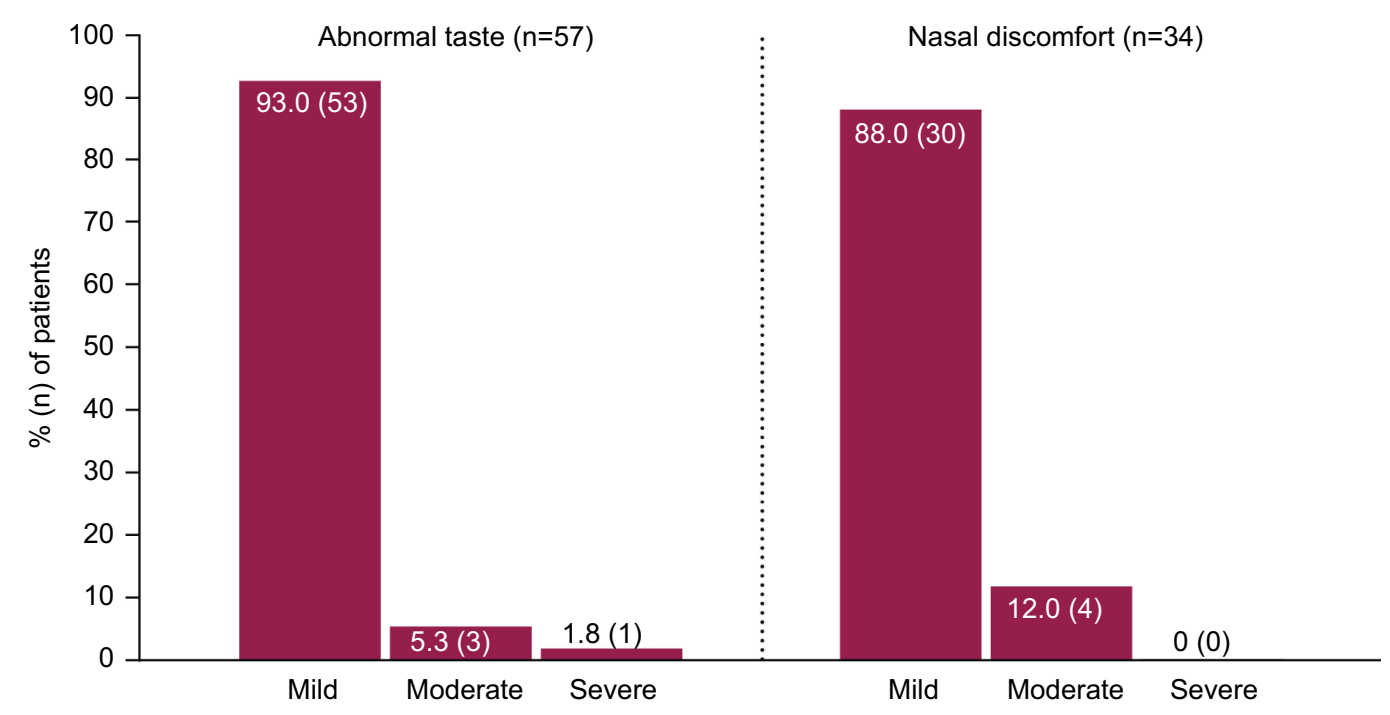

Figure 6 Severity of the most common treatment-emergent adverse events associated with AVP-825 in COMPASS

Notes: Data shown are for patients receiving AVP-825 + placebo tablet. During the other study treatment ( $100 \mathrm{mg}$ sumatriptan oral tablets + placebo delivery system), nine patients experienced abnormal product taste and three patients had nasal discomfort; all occurrences were mild in severity. Silberstein S, Winner PK, McAllister PJ, et al. Early onset of efficacy and consistency of response across multiple migraine attacks from the Randomized COMPASS Study: AVP-825 Breath Powered ${ }^{\circledR}$ Exhalation Delivery System (Sumatriptan Nasal Powder) vs Oral Sumatriptan. Headache. 2017;57(6):862-876. (c) 2017 American Headache Society. ${ }^{34}$ 
treatment, and a reduced risk of treatment-emergent nausea compared to sumatriptan oral. ${ }^{40}$

AVP-825 uptake in the clinic may be hampered by an assumption that it is "just another nasal spray", which may lead to concerns that AVP-825 is associated with many of the same disadvantages noted with traditional nasal sprays. While some medication is swallowed with any intranasal delivery method, AVP-825 was designed to reduce medication swallowing through the breath-powered closing of the soft palate. Pharmacokinetic data for AVP-825 as well as severity data for taste-related AEs in the AVP-825 COMPASS trial are consistent with this advantage. In COMPASS, 93\% of the cases of abnormal taste were classified as mild.

AVP-825 should be considered for patients who need rapid migraine relief and those who fail to tolerate triptans in oral, injectable, or conventional liquid nasal spray formulations. It may be especially useful in those with migraine with concurrent nausea or vomiting, patients who have difficulty in swallowing, and/or patients with gastroparesis or other GI problems. In addition, patients who have struggled with atypical sensations with higher doses of other triptan formulations may be ideal candidates for AVP-825. It should also be noted that migraine treatment is likely not going to be limited to AVP-825. Rather, AVP-825 may be an option along with other sumatriptan formulations for patients who use an attack-based care model to treat their migraines, ${ }^{19}$ in which the characteristics of an individual migraine attack dictate the use of a particular treatment.

Breath-powered technology has the potential to be adapted for other nasal and central nervous system-acting agents. A recently approved fluticasone propionate product for the treatment of nasal polyps employs this technology, ${ }^{23,41}$ and additional applications for this technology are under investigation. $^{42}$

\section{Key takeaways}

- The need for non-oral medication is clear in migraine, due to speed of some attacks and the presence of nausea, vomiting, and gastroparesis.

- The AEs associated with both SC and sumatriptan oral, the triptan atypical sensations, also suggest the need for an improved, more tolerable sumatriptan delivery system.

- The intense bitter taste of conventional liquid nasal sumatriptan discourages use.

- The development of a breath-powered dry nasal powder sumatriptan delivery system, AVP-825, was predicated on delivery of a lower dose of sumatriptan to the upper posterior of the nose for optimal absorption and a closure of the soft palate to reduce oral exposure and the bad taste.

- The comparative efficacy COMPASS trial, directly comparing the breath-powered dry nasal powder AVP825 system repeatedly to oral $100 \mathrm{mg}$ sumatriptan in a double dummy design, in the same patients and across multiple patients and attacks, demonstrated earlier onset of efficacy with a lower dose and fewer AEs for AVP825 over the oral standard of care, confirming the initial engineering and aligning the pharmacokinetics, anatomy, and drug presentation of the novel device with optimal outcomes for the acute treatment of migraine.

\section{Acknowledgment}

The authors thank Jennifer Hepker, PhD, from Prescott Medical Communications Group (Chicago, IL, USA) for editorial assistance.

\section{Disclosure}

Stewart J Tepper, MD, has consulted and/or participated in advisory boards for Acorda, Alder, Allergan, Amgen, ATI, Avanir Pharmaceuticals, Inc., Cefaly, Charleston Laboratories, DeepBench, Dr. Reddy's, electroCore, eNeura, Eli Lilly, GLG, Guidepoint Global, Impax, Pfizer, Scion Neurostim, Slingshot Insights, Supernus, Teva, and Zosano, received research support (no personal compensation, not tied to salary) from Alder, Allergan, Amgen, ATI, Avanir, Dr. Reddy's, electro-Core, eNeura, Scion Neurostim, Teva, and Zosano, holds stock options from ATI, receives salary from Dartmouth-Hitchcock Medical Center and the American Headache Society, and receives royalties from Springer. The authors report no other conflicts of interest in this work.

\section{References}

1. Headache Classification Committee of the International Headache Society (IHS). The International Classification of Headache Disorders, 3rd edition. Cephalalgia. 2018;38(1):1-211.

2. Marmura MJ, Silberstein SD, Schwedt TJ. The acute treatment of migraine in adults: the American Headache Society evidence assessment of migraine pharmacotherapies. Headache. 2015;55(1):3-20.

3. Loder E. Triptan therapy in migraine. $N$ Engl J Med. 2010;363(1): 63-70.

4. GBD 2016 Disease and Injury Incidence and Prevalence Collaborators. Global, regional, and national incidence, prevalence, and years lived with disability for 328 diseases and injuries for 195 countries, 1990-2016: a systematic analysis for the Global Burden of Disease Study 2016. Lancet. 2017;390(10100):1211-1259.

5. QuickStats: percentage of adults aged $\geq 18$ years who reported having a severe headache or migraine in the past 3 months, by sex and age group - National Health Interview Survey, United States, 2015. MMWR. 2017;66(24):654. 
6. Lipton RB, Fanning KM, Serrano D, Reed ML, Cady R, Buse DC. Ineffective acute treatment of episodic migraine is associated with new-onset chronic migraine. Neurology. 2015;84(7):688-695.

7. Becker WJ. Acute migraine treatment in adults. Headache. 2015;55(6): 778-793.

8. Diamond S, Bigal ME, Silberstein S, Loder E, Reed M, Lipton RB. Patterns of diagnosis and acute and preventive treatment for migraine in the United States: results from the American Migraine Prevalence and Prevention study. Headache. 2007;47(3):355-363.

9. Johnston MM, Rapoport AM. Triptans for the management of migraine. Drugs. 2010;70(12):1505-1518.

10. Silberstein SD. Practice parameter: evidence-based guidelines for migraine headache (an evidence-based review): report of the Quality Standards Subcommittee of the American Academy of Neurology. Neurology. 2000;55(6):754-762.

11. Camara-Lemarroy CR, Rodriguez-Gutierrez R, Monreal-Robles R, Marfil-Rivera A. Gastrointestinal disorders associated with migraine: a comprehensive review. World J Gastroenterol. 2016;22(36):8149-8160.

12. Tepper SJ, Rapoport AM. The triptans: a summary. CNS Drugs. 1999;12(5):403-417.

13. IMITREX (sumatriptan succinate) tablets, for oral use [Prescribing information]. GlaxoSmithKline Research Triangle Park, NC, USA; 2013.

14. Dixon CM, Saynor DA, Andrew PD, Oxford J, Bradbury A, Tarbit MH. Disposition of sumatriptan in laboratory animals and humans. Drug Metab Dispos. 1993;21(5):761-769.

15. Dodick DW. Triptan nonresponder studies: implications for clinical practice. Headache. 2005;45(2):156-162.

16. Gallagher RM, Kunkel R. Migraine medication attributes important for patient compliance: concerns about side effects may delay treatment. Headache. 2003;43(1):36-43.

17. Cady RK, Munjal S, Cady RJ, Manley HR, Brand-Schieber E. Randomized, double-blind, crossover study comparing DFN-11 injection (3 mg subcutaneous sumatriptan) with $6 \mathrm{mg}$ subcutaneous sumatriptan for the treatment of rapidly-escalating attacks of episodic migraine. J Headache Pain. 2017;18(1):17.

18. Derry CJ, Derry S, Moore RA. Sumatriptan (all routes of administration) for acute migraine attacks in adults - overview of Cochrane reviews. Cochrane Database Syst Rev. 2014;5:CD009108.

19. Silberstein SD, Marcus DA. Sumatriptan: treatment across the full spectrum of migraine. Expert Opin Pharmacother. 2013;14(12):1659-1667.

20. IMITREX (sumatriptan succinate) injection, for subcutaneous use [Prescribing information]. GlaxoSmithKline Research Triangle Park, NC, USA; 2015

21. Sumavel DosePro (sumatriptan injection), for subcutaneous use [Prescribing information]. Endo Pharmaceuticals Inc. Malvern, PA, USA; 2016.

22. ONZETRA ${ }^{\circledR}$ Xsail $^{\circledR}$ (sumatriptan nasal powder) [Prescribing information]. Avanir Pharmaceuticals, Inc. Aliso Viejo, CA, USA; 2016.

23. Djupesland PG. Nasal drug delivery devices: characteristics and performance in a clinical perspective - a review. Drug Deliv Transl Res. 2013;3(1):42-62.

24. Djupesland PG, Messina JC, Mahmoud RA. Breath powered nasal delivery: a new route to rapid headache relief. Headache. 2013;53(Suppl 2): 72-84.

25. Djupesland PG, Skretting A. Nasal deposition and clearance in man: comparison of a bidirectional powder device and a traditional liquid spray pump. J Aerosol Med Pulm Drug Deliv. 2012;25(5):280-289.
26. Fuseau E, Petricoul O, Moore KH, Barrow A, Ibbotson T. Clinical pharmacokinetics of intranasal sumatriptan. Clin Pharmacokinet. 2002;41(11):801-811.

27. Duquesnoy C, Mamet JP, Sumner D, Fuseau E. Comparative clinical pharmacokinetics of single doses of sumatriptan following subcutaneous, oral, rectal and intranasal administration. Eur J Pharm Sci. 1998;6(2):99-104.

28. Derry CJ, Derry S, Moore RA. Sumatriptan (intranasal route of administration) for acute migraine attacks in adults. Cochrane Database Syst Rev. 2012;2:CD009663.

29. Obaidi M, Offman E, Messina J, Carothers J, Djupesland PG, Mahmoud RA. Improved pharmacokinetics of sumatriptan with Breath Pow$\operatorname{ered}^{\mathrm{TM}}$ nasal delivery of sumatriptan powder. Headache. 2013;53(8): $1323-1333$

30. Luthringer R, Djupesland PG, Sheldrake CD, et al. Rapid absorption of sumatriptan powder and effects on glyceryl trinitrate model of headache following intranasal delivery using a novel bi-directional device. J Pharm Pharmacol. 2009;61(9):1219-1228.

31. Cady RK, McAllister PJ, Spierings EL, et al. A randomized, doubleblind, placebo-controlled study of breath powered nasal delivery of sumatriptan powder (AVP-825) in the treatment of acute migraine (The TARGET Study). Headache. 2014;55(1):88-100.

32. Djupesland PG, Docekal P; Czech Migraine Investigators Group. Intranasal sumatriptan powder delivered by a novel breath-actuated bi-directional device for the acute treatment of migraine: a randomised, placebo-controlled study. Cephalalgia. 2010;30(8):933-942.

33. Tepper SJ, Cady RK, Silberstein S, et al. AVP-825 breath-powered intranasal delivery system containing $22 \mathrm{mg}$ sumatriptan powder vs $100 \mathrm{mg}$ oral sumatriptan in the acute treatment of migraines (The COMPASS Study): a comparative randomized clinical trial across multiple attacks. Headache. 2015;55(5):621-635.

34. Silberstein S, Winner PK, McAllister PJ, et al. Early onset of efficacy and consistency of response across multiple migraine attacks from the Randomized COMPASS Study: AVP-825 Breath Powered ${ }^{\mathbb{R}}$ Exhalation Delivery System (Sumatriptan Nasal Powder) vs Oral Sumatriptan. Headache. 2017;57(6):862-876.

35. Davies GM, Santanello N, Lipton R. Determinants of patient satisfaction with migraine therapy. Cephalalgia. 2000;20(6):554-560.

36. Lipton RB, Hamelsky SW, Dayno JM. What do patients with migraine want from acute migraine treatment? Headache. 2002;42(Suppl 1):3-9.

37. Smelt AF, Louter MA, Kies DA, et al. What do patients consider to be the most important outcomes for effectiveness studies on migraine treatment? Results of a Delphi study. PLoS One. 2014;9(6):e98933.

38. Ferrari MD, Goadsby PJ, Roon KI, Lipton RB. Triptans (serotonin, 5-HT1B/1D agonists) in migraine: detailed results and methods of a meta-analysis of 53 trials. Cephalalgia. 2002;22(8):633-658.

39. Pierce M. Oral triptans and nausea: treatment considerations in migraine. Headache. 2013;53(Suppl 1):17-20.

40. Lipton RB, McGinley JS, Shulman KJ, Silberstein SD, Wirth RJ, Buse DC. AVP-825 (sumatriptan nasal powder) reduces nausea compared to sumatriptan tablets: results of the COMPASS Randomized Clinical Trial. Headache. 2018;58(2):229-242.

41. $\mathrm{XHANCE}^{\mathrm{TM}}$ (fluticasone propionate) nasal spray, for intranasal use [Prescribing Information]. Available from: https://www.xhance.com/ wp-content/themes/xhance/assets/pdf/XHANCE_Full_Prescribing_Information.pdf. 2017. Accessed February 27, 2018.

42. OptiNose US, Inc. Research \& Development. Available from: https:// www.optinose.com/research-and-development/overview-chart. 2017 Accessed February 27, 2018. 


\section{Publish your work in this journal}

Medical Devices: Evidence and Research is an international, peerreviewed, open access journal that focuses on the evidence, technology, research, and expert opinion supporting the use and application of medical devices in the diagnosis, monitoring, treatment and management of clinical conditions and physiological processes. The identification of novel devices and optimal use of existing devices which will lead to improved clinical outcomes and more effective patient management and safety is a key feature. The manuscript management system is completely online and includes a quick and fair peer-review system. Visit http://www. dovepress.com/testimonials.php to read real quotes from authors.

Submit your manuscript here: https://www.dovepress.com/medical-devices-evidence-and-research-journal 